\title{
SOME DATA ON SEGREGATION IN SACCHAROMYCES
}

\author{
URS LEUPOLD * and HÉLĖNE HOTTINGUER \\ Laboratoire de Génétique de la Foculté des Sciences \\ et Laboratoire de Génétique physiologique du C.N.R.S., \\ Paris
}

Received 14.ix.53

\section{INTRODUCTION}

Segregation in yeast has been described for many characters since Winge and Laustsen's first demonstration of segregation for morphological properties (1937). In 1949, Lindegren (1949a) presented the first chromosome maps of Saccharomyces. Frequently, the pairs of characters studied segregated in a $2: 2$ ratio within the yeast ascus, thus indicating simple, monohybrid inheritance (e.g. Pomper and Burkholder, I949).

In a number of instances, however, complex segregations were encountered. Some of these could readily be accounted for on the basis of multiple genes. $\dagger$ Thus, Lindegren and Lindegren (1947) were able to explain complex segregations for the formation of pink pigment as a result of the cooperation of two pairs of genes controlling requirements for adenine and methionine, respectively. Winge and Roberts (1948, 1950b, 1952) and Gilliland (1949) showed that simultaneous segregation of several polymeric genes was responsible for the $3: I$ and $4: 0$ (in addition to $2: 2$ ) ratios observed for ability to ferment maltose, raffinose and sucrose. Thorne (I95I) interpreted in a similar way segregations encountered in a study of the inheritance of flocculent growth.

On the other hand, a number of occasional deviations from the simple $2: 2$ ratio have been reported which are not easily explained in terms of multiple genes. For interpreting unexpected ratios obtained from crosses which usually give $2: 2$ segregations, orthodox mutation has been invoked now and then. An alternative hypothesis has been advanced by Winge and Roberts (1950a) who ascribed the observed deviations to a chance survival of four nuclei from an originally eight-nucleate complement during ascospore formation.

For interpreting two tetrads of Schizosaccharomyces pombe which segregated aberrantly for the alleles controlling homothallism and

* Present address : Institute of General Botany, University of Zurich, Zurich, Switzerland.

$\dagger$ The term "multiple gene segregation" will be used in this paper for describing simultaneous segregation of two or more pairs of alleles co-operating in the control of one pair of characters, regardless of the type of interaction (polymeric, complementary, etc.) involved. 
heterothallism in this yeast (see Leupold, 1950), an explanation in terms of a triploid or at least trisomic condition of the ascus mother cells was found necessary (Leupold, 1948, unpub.). Each tetrad appeared to segregate into two diploid and two haploid spores. The diploidy of some of the segregants was confirmed $(a)$ by analysing their haploid offspring, and $(b)$ by crossing one of the diploid segregants to a haploid clone, which led to segregations expected from a triploid. Roman, Hawthorne and Douglas (I95I), in reporting on a tetraploid segregation in Saccharomyces, have pointed out that from meiosis in polyploids, $4: 0,3:$ I, $2: 2, I: 3$ and (in the case of triploids, as a result of occasional loss of chromosomes) even $0: 4$ segregations may be expected. Polyploid segregations in Saccharomyces have further been described by Lindegren and Lindegren (I95I) and Pomper (I952).

Several known genetic mechanisms may thus result in complex segregations in yeast. However, convinced that they are not sufficient to account for many of the observed deviations from the $2: 2$ ratio, Lindegren (I949a, I 953) has in addition postulated " gene conversion", a mechanism which, by the transfer of genic material from one gene to its allele in the heterozygous condition, may endow the recessive gene with capacities of the dominant allele and vice versa. Though capable of explaining any complex segregation, this hypothesis is difficult to verify except by way of exclusion of strictly Mendelian interpretations. Of these, control by multiple genes, as well as chance survival of four nuclei from an originally eight-nucleate complement, have been ruled out as general explanations (Lindegren, I949a; Mundkur, I950). In Mundkur's (1949) study of aberrant segregations for galactose and melibiose fermentation, the intervention of orthodox mutations as a possible cause of deviations from the expected ratios was minimised by reducing the time elapsing between germination of the ascospores and application of the fermentation test. Mundkur did not exclude, however, polyploidy as a possible mechanism causing the observed deviations from the $2: 2$ ratio, since (as pointed out by Roman and co-workers) not only $3: 1$ and $4: 0$, but also $I: 3$ and even $0: 4$ segregations may be expected from polyploids.

It is the purpose of this paper to present some further data on segregation in Saccharomyces cerevisiae. These data were obtained in the course of work on the spontaneous formation of the mutant "petite colonie" (Ephrussi and Hottinguer, I95I), as a by-product of crosses designed to introduce a number of marker genes into our stocks.

\section{MATERIAL AND METHODS}

\section{(a) Strains and markers}

The following marker pairs were introduced into our stocks : Independence of dependence on : histidine (HIS/his), tryptophane (TRY/try), methionine (MET) $m e t)$, uracil $(U R / u r)$, and adenine $(A D / a d)$; ability/inability to ferment galactose 
$(G / g)$; and disperse/flaky growth in liquid yeast extract-glucose medium $(D / d)$.* The mating type alleles, $a$ and $\alpha, \dagger$ were used as additional markers. These marker pairs were derived from the following strains :

I6o $(\alpha), B_{I 5}(a)$ and $868 / 3 b(\alpha)$ (HIS TRY MET UR AD $g$; haploid descendants of "Boulangerie II ", a diploid obtained from the Pasteur Institute in Paris).

${ }^{I 75} / 6 b$ p $p_{1}$ (a HIS TRY MET UR ad G ; haploid descendant of "Yeast Foam" (see Ephrussi, Hottinguer and Tavlitzki, 1949);

$R 2$ ( $\alpha$ his try MET UR AD G; a haploid obtained from Dr H. Roman).

$P_{3}$ (a $\alpha I S H I S T R Y$ try MET met UR ur AD AD GgDd; a diploid obtained from Dr S. Pomper, originally prepared by crossing : $~ H I S T R Y$ met UR $A D G d \times a$ HIS try $M E T$ ur $A D g D)$.

$L-4 I / 2 d$ ( $a$ his try MET UR $A D g$; haploid segregant of $\left(B_{15} \times R 2\right)$ ).

$L-167 / 3 a$ ( $\alpha$ his try MET UR $A D g$; haploid segregant of $(868 / 3 b \times L-4 I / 2 d)$ ).

$P_{3 / 5 a}(a d)$ and $P_{3 / 1} I_{4}(\alpha D)$ (HIS TRY met ur $A D G$; haploid segregants of $P_{3) .}$

\section{(b) Determinations of mating type, growth factor requirements and ability to ferment galactose}

Mating type is determined by mixing two drops of a $24-48$ hour liquid culture of the strain to be tested with two drops of a $24-48$ hour liquid culture of a test strain of known mating type. The mixture is added to $2 \mathrm{ml}$. of liquid yeast extract medium (Difco Yeast Extract 0.5 per cent., glucose 3 per cent.) in a test tube and incubated for 24 hours at $25^{\circ} \mathrm{C}$.

Requirements for histidine, tryptophane, methionine and uracil are determined by streaking the strains to be tested on solid synthetic media, designated as " histidine free ", " tryptophane free ", " methionine free ", " uracil free ", and " complete". The complete medium is based on Wickerham's formula (Wickerham, 1946; Wickerham and Burton, 1948), but differs from it in that six of its growth factors are replaced by adenine and uracil. The formula for $1000 \mathrm{ml}$. of "complete" medium is the following :

$\mathrm{KH}_{2} \mathrm{PO}_{4}: 1 \cdot 0$ g. $\mathrm{MgSO}_{4} \cdot 7 \mathrm{H}_{2} \mathrm{O}: 0.5$ g. $\mathrm{NaCl}: 0.1$ g. $\mathrm{CaCl}_{2} \cdot 2 \mathrm{H}_{2} \mathrm{O}$ : o. I g. $\left(\mathrm{NH}_{4}\right)_{2} \mathrm{SO}_{4}: 3.5 \mathrm{~g}$. L-Asparagine : $1.5 \mathrm{~g}$. Glucose : 10.0 g.

$\mathrm{H}_{3} \mathrm{BO}_{3}: 500 \gamma$. CuSO $4.5 \mathrm{H}_{2} \mathrm{O}: 40 \gamma$. KI : $100 \gamma . \mathrm{FeCl}_{3} \cdot 6 \mathrm{H}_{2} \mathrm{O}: 200 \gamma$. $\mathrm{MnSO}_{4} \cdot 1 \mathrm{H}_{2} \mathrm{O}: 400 \gamma \cdot \mathrm{Na}_{2} \mathrm{MoO}_{4} \cdot 2 \mathrm{H}_{2} \mathrm{O}: 200 \gamma \cdot \mathrm{ZnSO}_{4} \cdot 7 \mathrm{H}_{2} \mathrm{O}: 400 \gamma$.

Biotin : $2 \gamma$. Calcium pantothenate : $400 \gamma$. Thiamine hydrochloride : $400 \gamma$. L-Histidine monohydrochloride. $\mathrm{H}_{2} \mathrm{O}$ : $10 \mathrm{mg}$. DL-Tryptophane: $20 \mathrm{mg}$. DL-Methionine : $20 \mathrm{mg}$. Uracil : $20 \mathrm{mg}$. Adenine : $20 \mathrm{mg}$.

Difco Bacto Agar : $20 \mathrm{~g}$.

The media "histidine free", "tryptophane free", etc. differ from the " complete" medium in that they lack histidine, tryptophane, etc., respectively.

Cell material for the growth factor tests is obtained from streak cultures on yeast extract agar. One small loopful of cell material is suspended in $10 \mathrm{ml}$. of Ringer's solution, and one large loopful of the suspension is streaked on a sector of a plate containing the desired medium. Each clone is inoculated on at least two media, namely, on the medium lacking the particular growth factor which the strain is tested for, and on "complete" medium which serves as a control. The plates are incubated at $25^{\circ} \mathrm{C}$., and the growth on the two media during the following five days is compared.

The adenine requirement is inferred from pigment formation on yeast extract agar containing 8 per cent. glucose. Under these conditions, all adenineless strains (including "petites" which might be present among the segregants; Tavlitzki, 1951) form a red pigment.

* See Pomper and Burkholder, 1949.

$\dagger$ The symbols a/ $\alpha$ used in this paper are those introduced by Lindegren and adopted also by Pomper and by Roman. Mating types $a$ and $\alpha$ correspond respectively to mating types + and - of Ephrussi. 
Ability to ferment galactose is determined on the basis of gas formation in Durham tubes. Each strain is tested in three media : No. 1 : 0.5 per cent. Difco yeast extract ; No. 2 : 0.5 per cent. yeast extract +2 per cent. glucose ; and No. 3 : 0.5 per cent. yeast extract +2 per cent. galactose. Media No. 1 and 2 serve as controls. A large loopful of cells taken from a streak culture on glucose-yeast extract agar is suspended in $2 \mathrm{ml}$. of Ringer's solution. From the suspension, three drops are inoculated into a test tube containing $5 \mathrm{ml}$. of medium, part of which fills a small, inverted test tube. The tubes are incubated at $25^{\circ} \mathrm{C}$. Strains which do not fill the inverted tube with gas within one week are classified as non-fermenters $(\mathrm{g})$, all other strains.as fermenters $(G)$.* $^{*}$

The classification for disperse/flaky growth $(D / d)$ is based on macroscopic growth habit in liquid yeast extract-glucose medium (Difco yeast extract 0.5 per cent., glucose 3 per cent.; cultures incubated at $25^{\circ} \mathrm{C}$.).

\section{(c) Technique of crossing}

Crosses are set up as mass combinations of cells of the two strains to be crossed, in the way described for the mating type tests. One day after the cultures have been combined, a loopful of the mixture is transferred to fresh yeast extract medium, and this is repeated on the second, third and fourth day ; each time, the inoculum is taken from the latest culture. After five days, the culture contains mainly diploid cells. A cell of typical diploid appearance is then isolated with a micromanipulator, and the clone to which it gives rise is transferred to yeast extract agar and therefrom to sporulation medium. Sporulation is induced on Gorodkowa agar or on Adams agar (Adams, 1949). In either case, care is taken to reduce presporulation growth to a few days in order to avoid intervening sporulations as much as possible.

\section{RESULTS}

(a) Segregation data

The data presented below describe segregations in six hybrids heterozygous for various markers, as follows :
1. $C L-4 I$ (B I $5 \times \mathrm{R}$ 2) (aa HIS his TRY try $G g$ ) (1 2 asci)
2. $C L-167(868 / 3 \mathrm{~b} \times \mathrm{L}-4 \mathrm{I} / 2 \mathrm{~d})$ (a a HIS his TRY try) (3 asci)
3. Cg6g (L-167/3a $\left.\times 175 / 6 \mathrm{~b} \quad \mathrm{p}_{1}\right)$ (a a HIS his TRY try $A D$ ad $G g)$ (3I asci)
4. $P_{3}$ ( a a TRY try MET met UR ur $\left.G g D d\right)$ (26 asci)
5. $C_{948}\left(\mathrm{P}_{3} / 5^{\mathrm{a}} \times 160\right)$ ( a a MET met UR ur $G \mathrm{~g} D d$ ) (29 asci)
6. $C 967\left(\mathrm{P}_{3} / \mathrm{r}_{4} \mathrm{c} \times \mathrm{r}_{75} / 6 \mathrm{~b}\right.$ p $\left.\mathrm{p}_{1}\right)$ (a a MET met UR ur $A D$ ad) (33 asci)

The numbers of analysed four-spored asci in which all four spores germinated are indicated in parentheses for each hybrid.

The frequencies of various segregation types observed in each hybrid are indicated in table $\mathrm{I}$. It is seen that a majority of $2: 2$ segregations was obtained for mating type (132 out of 134 asci); for ability to synthesise histidine $(44 / 46)$, tryptophane $(65 / 72)$, uracil $(87 / 88)$ and adenine $(62 / 64)$; and for growth habit (disperse/flaky) $(53 / 55)$. $2: 2$ segregations were somewhat less frequent for the ability to grow in the absence of methionine $(75 / 88)$ and for the

* The authors wish to thank Dr B. Ephrussi for performing all fermentation tests. 


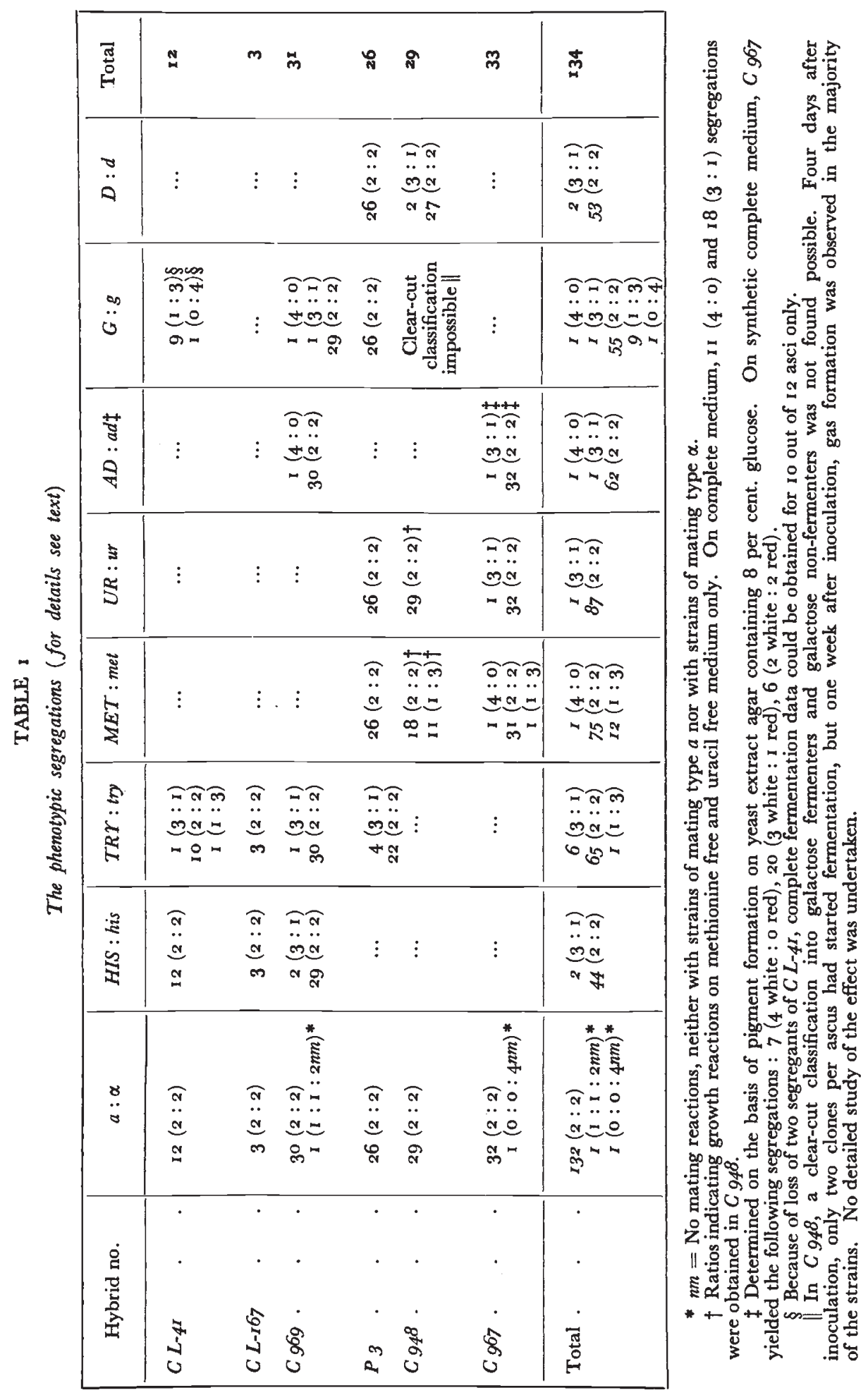


ability to ferment galactose $(55 / 67$, not counting 29 asci of $C 948$ in which the $2: 2$ ratio was frequently obscured by the appearance of slow fermenters).

The deviations from the $2: 2$ ratio which have been observed (see table I) may be classified as follows :

(i) Systematic deviations, occurring in an important fraction of the asci obtained from one hybrid and concerning one particular pair of characters only. This group includes the segregations for ability to ferment galactose in hybrid $C L-4 I$ (no $2: 2$ segregation among ro asci), for pigment formation on synthetic medium in hybrid $C_{967}$ (only 6 $2: 2$ segregations among 33 asci), and for growth on methionine free and complete medium in hybrid $C 94^{8}$ (on methionine free medium, only $182: 2$ segregations among 29 asci). In each of these cases, the observed deviations are probably due to simultaneous segregation of multiple genes controlling the pair of characters concerned. For a more detailed discussion, the reader is referred to paragraph $3 c$.

(ii) Systematic deviations, occurring in a few asci only but concerning most or all pairs of characters involved. Two clear-cut cases of this kind have been observed, namely, ascus 27 of hybrid $C 967$ (deviations for all characters involved : 4 non-maters, $3 A D:$ I ad, $4 M E T$ : o met, $3 U R: \mathrm{I} u r$ ), and ascus 16 of hybrid $C g 69$ (deviations for four out of the five characters involved : $2 a: 2 a, 4 A D: 0 a d, 3 H I S:$ I his, $3 T R Y:$ I try, $4 G: \circ g$ ). In both cases, the observed segregations could be shown to be due to polyploidy of the ascus mother cell concerned (Leupold, in press ; for main results, see paragraph $3^{d}$ ). The same interpretation is suggested in the case of a third ascus, $C 969 / 5$, by the presence of two non-maters (in addition to one $a$ and one $a$ clone) among the four single spore strains, combined with a $3:$ I segregation for histidine dependence. This ascus was not studied in detail.

(iii) Occasional deviations, occurring in a few asci and concerning one pair of characters per ascus only (in several cases, however, coinciding with systematic deviations of type (i) concerning a second pair of characters). Occasional deviations from the $2: 2$ ratio have been encountered for several marker pairs, as follows :

Hybrid $C L-4 I:$ I (3 $T R Y:$ I try) and I (I $T R Y: 3$ try).

Hybrid $C 969:$ I (3 $G:$ I $g$ ). Hybrid $\mathrm{P}_{3}: 4$ (3 $T R Y:$ I $\left.t r y\right)$.

Hybrid $C 94^{8}: 2$ (3 $D:$ I $\left.d\right)$. Hybrid $C 967$ : I (I $M E T: 3$ met).

No detailed study of these asci has been undertaken. Among several interpretations which might account for the observed segregations, the possibility of mutation should especially be taken into consideration.

\section{(b) Linkage relations}

On the basis of the segregations described above, an attempt was made to determine the linkage relations between the genes involved. Only asci segregating 2:2 for all characters concerned were taken 
as the basis for the calculations, with the following exceptions : (i) The asci obtained from the hybrids $C L-4 I$ and $C 94^{8}$ were excluded from the calculations only as far as linkage between the ability to ferment galactose and the other characters was concerned. (ii) In the case of the I I asci of $C 94^{8}$ which showed deviating segregations for the ability to grow on methionine free medium, the calculations were based on the interpretations given in paragraph $3 c$.

The cross $A B \times a b$ leads to three different types of asci : Type I (ditype) : $2 A B: 2 a b$. Type II (ditype) : $2 A B: 2 a b$. Type III (tetratype) : I $A B:$ I $A b:$ I $a B:$ I $a b$. Independent segregation of the two loci is indicated by equal frequencies of type I and II, linkage by type II being considerably less frequent than type I. If the two loci segregate independently of each other and one or both loci segregate independently of their centromeres, a ratio of I : II : III = I $:$ I $: 4$ is expected. Relative frequencies of type III asci lower than $2 / 3$, combined with equal frequencies of types I and II, suggest independence between the two loci but close linkage between each locus and its centromere.

In unordered tetrads (like those of Saccharomyces), in which the products of meiosis are not linearly arranged, the distance from the centromere cannot be determined directly for one particular locus. It is possible, however, to calculate the proportions of second-division segregation for three independent loci if the relative frequencies of tetratype asci are known from each of the three possible crosses, $A B \times a b$ (or $A b \times a B$ ), $A C \times a c$ (or $A c \times a C$ ), and $B C \times b c$ (or $B c \times b C$ ) (Perkins, 1949, and Whitehouse, 1950; a graphical method was developed by Lindegren, 1949a). The frequencies of second-division segregations so obtained may finally be converted into ordinary recombination frequencies (Spiegelman, 1952; Papazian, 1952).

Table 2 gives the frequencies of the three tetrad types for each pair of loci studied. Data from different crosses involving each pair of characters, whether in coupling or repulsion, are lumped together. On the other hand, data concerning tryptophane dependence have been separated according to the origin of the strains introducing tryptophane deficiency. This character has been derived partly from material obtained from $\mathrm{Dr} \mathrm{H}$. Roman (strain $R 2$ ) and partly from material supplied by $\operatorname{Dr} S$. Pomper (strain $P_{3}$ ). Since the corresponding genes are not necessarily identical, they are designated with the symbols $T R r_{R} / t r y_{R}$ and $T R Y_{P} / t r y_{P}$, respectively. The same holds for the segregation data involving inability to ferment galactose, a character which was partly derived from strains isolated in this laboratory (strain $160, B I_{5}$ and $868 / 3 b$; descendants of strain "Boulangerie II") and partly from strain $P_{3}$ which was received from Dr Pomper. The corresponding genes, which again need not be identical, are referred to by the symbols $G_{E} / g_{E}$ and $G_{P} / g_{P}$ respectively.

In the third column of table $2, \chi^{2}$ and $\mathrm{P}$ are given on the assumption that the observed numbers of type I and II tetrads represent random 
samples of a population in which the two types are equally frequent. It can be seen that close linkage is indicated only for HIS/his and $T R r_{R} / \operatorname{try}_{R}(\mathrm{P}<\mathrm{0} .00 \mathrm{I}$, recombination value $=19.5$ per cent. $)$. It should be mentioned, however, that thus far only part of the possible gene combinations have been realised.

In the fourth column of table $2, \chi^{2}$ and $\mathrm{P}$ are calculated on the assumption that the observed frequencies of the three tetrad types were obtained as random samples from a population in which the

TABLE 2

Linkage relations. (For explanation, see text)

\begin{tabular}{|c|c|c|c|c|c|c|c|}
\hline \multirow[t]{2}{*}{ Cross } & \multicolumn{3}{|c|}{$\begin{array}{l}\text { Frequency of } \\
\text { tetrad types }\end{array}$} & \multicolumn{2}{|c|}{$\begin{array}{c}\chi^{2} \text { and } \mathrm{P} \text { for } \\
I: I I=\text { sample } \\
\text { from basic ratio } \\
I: I\end{array}$} & \multicolumn{2}{|c|}{$\begin{array}{l}\chi^{2} \text { and } P \text { for } \\
\text { I }: \text { II }: \text { III }=\text { sample } \\
\text { from basic ratio } \\
\quad I: I: 4\end{array}$} \\
\hline & I & II & III & $x^{2}$ & $\mathbf{P}$ & $x^{2}$ & $\mathbf{P}$ \\
\hline$a \times H I S$ & 9 & 10 & 22 & 0.05 & 0.8 & $3 \cdot 20$ & 0.2 \\
\hline$a \times T R r_{R}$ & 5 & II & 25 & $2 \cdot 25$ & 0.1 & 3.23 & 0.2 \\
\hline$a \times T R r_{P}$ & 6 & 5 & II & 0.09 & 0.8 & $2 \cdot 86$ & 0.2 \\
\hline$a \times M E T$. & 12 & 17 & 53 & 0.86 & 0.4 & $r \cdot 09$ & $0 . \overline{6}$ \\
\hline$a \times U R \quad$. & 15 & 17 & 50 & 0.13 & 0.7 & $1 \cdot 35$ & 0.5 \\
\hline$a \times A D$ & II & II & 37 & 0 & I. & 0.39 & 0.8 \\
\hline$a \times G_{E}$ & 7 & 2 & 19 & $2 \cdot 78$ & $0 \cdot 1$ & $2 \cdot 70$ & 0.3 \\
\hline$a \times G_{P}$ & I & 6 & 15 & $3: 57$ & 0.05 & 3.43 & 0.2 \\
\hline$a \times D$ & 7 & 7 & 35 & 0 & $1 \cdot 0$ & 0.51 & 0.8 \\
\hline$H I S \times T R r_{R}$ & 25 & o & 16 & $25 \cdot 0$ & $<0.001$ & $\ldots$ & \\
\hline$H I S \times A D$ & 4 & 5 & 19 & $O \cdot I I$ & 0.7 & $0.4 I$ & 0.8 \\
\hline$H I S \times G_{E}$ & 3 & $\widehat{6}$ & 19 & $1 \cdot \infty$ & 0.3 & 0.90 & 0.6 \\
\hline$T R r_{R} \times A \dot{D}$ & 4 & 5 & 19 & $0 \cdot 11$ & 0.7 & $0.4 I$ & 0.8 \\
\hline$T R r_{R} \times G_{E}$ & 3 & 7 & 18 & $1 \cdot 60$ & 0.2 & 1.76 & 0.4 \\
\hline$T R r_{P} \times M E T$ & 4 & 4 & 14 & & $I \cdot 0$ & 0.09 & 0.95 \\
\hline$T R r_{P} \times U R$ & I & 4 & 17 & $1 \cdot 80$ & 0.2 & $2 \cdot 34$ & 0.3 \\
\hline$T R r_{P} \times G_{P}$ & 2 & 6 & 14 & $2 \cdot 00$ & 0.2 & $2 \cdot 27$ & 0.3 \\
\hline$T R r_{P} \times D$ & I & 4 & 17 & $1 \cdot 80$ & 0.2 & $2 \cdot 34$ & \\
\hline$M E T \times U R$ & 14 & 13 & 55 & 0.04 & 0.9 & 0.04 & 0.98 \\
\hline$M E T \times A D$ & 6 & 7 & 18 & 0.08 & 0.8 & 0.60 & 0.7 \\
\hline$M E T \times G_{P}$ & 2 & 4 & 16 & 0.67 & 0.4 & $0.9 I$ & 0.6 \\
\hline$M E T \times D$ & 10 & 10 & 29 & 0 & $1 \cdot 0$ & $1 \cdot 23$ & 0.5 \\
\hline$U R \times A D$ & 6 & 7 & 18 & 0.08 & 0.8 & 0.60 & 0.7 \\
\hline $\begin{array}{l}U R \times G_{P} \\
U R \times D\end{array}$ & 4 & $\begin{array}{l}2 \\
0\end{array}$ & $\begin{array}{l}16 \\
29\end{array}$ & $\begin{array}{l}0.67 \\
0.20\end{array}$ & 0.4 & 0.91 & $0 \cdot 6$ \\
\hline $\begin{array}{l}U R \times D \\
A D \times G_{E}\end{array}$ & $\begin{array}{r}11 \\
3\end{array}$ & $\begin{array}{l}9 \\
3\end{array}$ & $\begin{array}{l}29 \\
22\end{array}$ & $\begin{array}{l}0.20 \\
0\end{array}$ & $\begin{array}{l}0.7 \\
1 \cdot 0\end{array}$ & $\begin{array}{l}1 \cdot 47 \\
1 \cdot 79\end{array}$ & $\begin{array}{l}0.5 \\
0.4\end{array}$ \\
\hline$G_{P} \times D^{L}$. & 4 & 3 & 15 & 0.14 & 0.7 & 0.16 & 0.9 \\
\hline
\end{tabular}

ratio I : II : III was I $: I: 4$. It is obvious that in no case the observed deviations from the basic ratio are sufficiently important to suggest close linkage between a locus and the centromere. Precise calculations following the method of Whitehouse would therefore be of little value. The absence of ratios deviating significantly from the I : I : 4 ratio does not exclude, however, close linkage of at least some loci with their respective centromeres, for two reasons : (i) larger samples might have revealed significant deviations which remained 
undetected with the small samples actually studied; (ii) tetratype frequencies smaller than $2 / 3$ are obtained only in cases where both genes are closely linked to their centromere; it is not excluded, therefore, that at least one of the genes (or any two independent genes the simultaneous segregations of which have not yet been studied) were closely associated with the centromere.

It is not possible, from these data, to decide whether the two genes for tryptophane deficiency, $t r y_{R}$ and $t r y_{P}$, are identical. The same applies to the two genes for inability to ferment galactose, $g_{E}$ and $g_{p}$.

Lindegren's segregation data involving mating type, methionine dependence, adenine dependence and galactose fermentation (Lindegren, I949a) agree with our results concerning the same characters in as far as they suggest absence of linkage between the loci involved. However, pairwise comparison of the frequencies of tetratype asci obtained in the crosses $a \times M E T, a \times A D, a \times G_{E}, a \times G_{p}$, $M E T \times A D, M E T \times G_{P}$ and $A D \times G_{E}$, with the corresponding tetratype frequencies reported by Lindegren for his gene pairs $a / a$, $M T / m t, A D / a d$ and $G / g$, reveals a significant difference in the combination $A D \times G_{E}$ (Lindegren's data : $45 / 147$ in the cross $A D \times G$; our data: $22 / 28$ in the cross $\left.A D \times G_{E} ; \mathrm{P}<0.001\right)$. It cannot be decided, on the basis of these comparisons, whether our genes $a$, $m e t$ and $g_{p}$ are identical with the corresponding genes $a, m t$ and $g$ described by Lindegren. It is probable, however, that of the two genes $g_{E}$ and $a d$, at least one is different from the corresponding genes $g$ and ad present in Lindegren's material. Our results are in agreement with the assumption of independent segregation between at least one of the two loci and its centromere, whereas Lindegren's data indicate close linkage between each of the two loci and their centromeres (approximate distances from centromere : 6 units for $g$ and I I units for $a d$, determined by including tetratype frequencies which involve $a / a$ as a third locus).

Such differences are not surprising, since the genes $g$ and $a d$ described by Lindegren and the corresponding genes used in our work have different origins. In the case of galactose fermentation it is even probable, from the results described in the following paragraph, that more than one gene is involved in the control of this character.

\section{(c) Multiple gene segregations}

Galactose.-Out of ten asci obtained from hybrid $C L-4 I$, nine contained one galactose fermenter and three non-fermenters, and one ascus yielded four non-fermenters. This suggests that two complementary genes controlling the fermentation of galactose are involved in cross $\left(B_{I 5} \times R_{2}\right)$, and that ability to ferment this sugar requires the simultaneous presence of the two dominant genes. Since 
$R 2$ is a galactose fermenter, the two recessive genes have been introduced into the cross presumably by $B{ }_{15}$.

These results represent the first case suggesting a complementary instead of a polymeric control of sugar fermentation. A biochemical basis for a complementary mechanism of control may be found in the results of Leloir and his colleagues (Trucco and co-workers, $194^{8}$; Caputto and co-workers, 1949, 1950 ; Cardini and co-workers, 1949), who showed that at least three enzymatic steps are involved in the conversion of galactose into an intermediate which is common to the glucozymase complex. Each step is catalysed by an enzyme which in turn may be assumed to be controlled by a gene. If the

TABLE 3

Segregation in hybrid $\mathrm{C} 948$ : The three types of segregation for ability to grow on methionine free, uracil free and complete medium, represented by three typical asci

\begin{tabular}{|c|c|c|c|c|}
\hline \multirow{2}{*}{ Strain no. } & \multicolumn{3}{|c|}{ Growth on: } & \multirow{2}{*}{ Genotype } \\
\hline & Methionine free & Uracil free & Complete & \\
\hline $\begin{array}{r}14 a \\
b \\
c \\
d\end{array}$ & $\frac{ \pm}{+}$ & $\frac{\bar{t}}{\bar{t}}$ & $\begin{array}{l}+ \\
+ \\
+ \\
+\end{array}$ & $\begin{array}{l}M E T \text { ur } C \\
\text { met } U R C \\
\text { met ur } C \\
M E T U R C\end{array}$ \\
\hline $\begin{array}{r}13 a \\
b \\
c \\
d\end{array}$ & $\frac{ \pm}{t}$ & $\begin{array}{l}+ \\
+ \\
-\end{array}$ & $\frac{+}{+}$ & $\begin{array}{l}M E T U R \\
\text { met } U R \\
\text { met ur } \\
M E T \text { ur } C\end{array}$ \\
\hline $\begin{array}{r}15 a \\
b \\
c \\
d\end{array}$ & $\underline{-}$ & $\frac{ \pm}{ \pm}$ & $\begin{array}{l}+ \\
+ \\
+\end{array}$ & $\begin{array}{l}\text { met } U R \\
M E T \text { ur } \\
M E T U R \\
\text { met ur } C\end{array}$ \\
\hline
\end{tabular}

formation of each of the three enzymes required the presence of the dominant at one of the corresponding three loci, then a hybrid heterozygous for two or three genes would be expected to yield $2: 2, \mathrm{I}: 3$ and $0: 4$ segregations. Of these ratios, the latter two have actually been encountered in $C L-4 I$, and the absence of tetrads yielding a $2: 2$ ratio may be due to the small number of asci studied.

Adenine, methionine and pigment formation.-In hybrid $C 967$, regular segregation for the ability to synthesise adenine was shown by $2: 2$ segregations for the formation of red pigment. This, however, was true only for the cultures which were grown on yeast extract agar containing 8 per cent. glucose; one exceptional $3:$ I segregation (ascus $C 967 / 27$ ) will be discussed in paragraph $3 d$. On "complete" synthetic medium, pigment formation was found to depend on 
methionine as well as on adenine dependence, for the recombinants carrying both recessives remained white. This led to 4 : o segregations (7 ditype asci containing the two recombinant genotypes : $A D M E T$ and ad met) and $3:$ I segregations ( $18 *$ tetratype asci containing I $A D M E T:$ I $A D$ met : I ad met : I ad $M E T$ ) in addition to $2: 2$ segregations for white and red (6 ditype asci yielding the two parental genotypes : $A D$ met and ad $M E T)$. This dependence of pigment formation by adenineless clones on the ability to synthesise methionine has been reported earlier by Lindegren and Lindegren (1947).

Uracil.-A co-operation of two pairs of genes is also probable in the complex segregations observed in hybrid $C_{948}$. Comparison of the results of growth factor tests carried out on methionine free, uracil free and "complete" medium revealed the existence of three types of asci. Table 3 gives the results for one typical ascus of each group. In I I out of 29 asci, $2: 2$ segregation for the ability to grow on methionine free medium was accompanied by the ability of all four segregants to grow on complete medium. In a second group of 7 asci, $2: 2$ segregation was observed on methionine free medium but one of the two methionineless segregants of each ascus failed to grow on complete medium. A last group of I I asci segregated I : 3 on methionine free and $3:$ I on complete medium; it was always one of the three methionine-negative segregants which failed to grow on complete medium. The I 8 aberrant clones which were unable to utilise the two media were also unable to grow on uracil free medium. Since, on the latter, $2: 2$ segregation was observed in all 29 asci, it was concluded that all aberrant segregants carried the gene for uracil deficiency.

A possible interpretation of these findings is as follows. It may be assumed that the gene "ur" present in the strain $P_{3} / 5 a$ (one of the parents of hybrid $C_{948}$ ) blocks the synthesis of an unknown growth factor $\mathrm{X}$ rather than of uracil. However, due to the presence of a gene $C, P_{3} / 5 a$ is able to utilise exogeneous uracil which it converts into X. Strain 160 (the other parent of hybrid $\left.C_{948}\right)^{\text {) is unable to }}$ carry out this conversion because it contains the allele $c$. On the other hand, it is independent of uracil since the presence of the gene " UR" permits direct synthesis of the growth factor $\mathrm{X}$. The two loci are linked. In tetrads which have not undergone crossing-over between the two loci, the two parental genotypes $U R c$ and $u r C$ will segregate in a $2: 2$ ratio. Phenotypically, this results in two segregants being able to grow both on uracil free and on complete medium, and in two segregants which grow on the latter but not on the former medium. In tetrads in which one crossing-over has taken

* Excluded are two asci in which the $3: 1$ segregation for pigment formation was combined with deviations in the segregation of $A D / a d$ and $M E T /$ met, namely, with a $1: 3$ segregation for $M E T / m e t$ in ascus $C 967 / 23$, and with $3: 1$ and $4: 0$ segregations for $A D / a d$ and $M E T /$ met, respectively, in ascus $C$ g67/27 (see paragraph $\left.3^{d}\right)$. In both cases, pigment formation on synthetic medium was confined to strains combining dependence on adenine with independence of methionine. 
place between the two loci, two recombinant genotypes are obtained in addition to the two parental ones, as follows :

$\begin{array}{lccccc} & U R C & U R C & \text { ur } C & \text { ur } c \\ \text { Growth on uracil free medium } & \cdot & + & + & \circ & \circ \\ \text { Growth on complete medium } & \cdot & + & + & + & \circ\end{array}$

Of the two recombinant types, one is able to manufacture $\mathrm{X}$ directly as well as to convert uracil into $\mathrm{X}$, whereas the other one has lost both activities. It is this last type of strains which does not grow even on "complete" medium since the hypothetical growth factor $\mathrm{X}$ is not available in any of the synthetic media used. This factor must be present in yeast extract, however, since growth on medium containing yeast extract is normal.

Four strand double crossing-over between the two loci in question would correspondingly result in a $2: 2$ segregation for the two recombinant genotypes. Phenotypically, this would lead to $2: 2$ segregations both on uracil free and on complete medium. The rarity of double crossing-overs is probably responsible for the fact that this last type of segregation has not been observed in our material.

Segregation on methionine free medium is disturbed only in tetrads in which recombination has taken place and in which the recombinant genotype urc happens to be combined with $M E T$. If we assume that in every one of the $I$ I asci in which $I: 3$ segregation has been obtained on methionine free medium, the one segregant which does not grow on any medium is methionine positive, segregation for $M E T / m e t$ is perfectly regular.

In the last column of table 3 , the interpretation given to the phenotypical segregations is indicated for three typical asci. As to the alleles $C / c$, complete interpretation is only possible for asci in which the parental genotypes $U R C$ and $u r C$ have segregated in a $2: 2$ ratio. In all other asci, $C$ and $c$ can be distinguished in the uracil negative but not in the uracil positive segregants; it is to be predicted, however, that in these tetrads, one of the uracil positive clones carries $C$, the other $c$.

\section{(d) Polyploid segregations}

From hybrid $C 967$, an ascus was isolated in which the four characters involved segregated as follows :

\begin{tabular}{rllll} 
Strain & Mating type & \multicolumn{3}{c}{ Synthesis of } \\
no. & no mating reactions & Ad & Met & Ur \\
$27 a:$ & no mating reactions & + & + & - \\
$27 b:$ & no & + & + \\
$27 c:$ & no mating reactions & - & + & + \\
$27 d:$ & no mating reactions & + & + & +
\end{tabular}

On the basis of results obtained in Schizosaccharomyces pombe (Leupold, 1948, unpublished) and of the findings of Roman and co-workers (195I) and of Lindegren and Lindegren (I95I) in Saccharomyces 
cerevisiae, these segregations can be interpreted as resulting from meiosis in a polyploid. Indeed, the four clones isolated from the ascus not only formed cells of size and shape characteristic of diploids, but were also able to sporulate. Analysis of the progeny confirmed the diploidy of the four strains. Twelve asci, three from each of the four clones, were dissected, and the segregations for the four characters involved were studied. The $4: 0,2: 2$ and $0: 4$ ratios which were obtained indicated that the spores of ascus 27 had the following constitution :

$\begin{array}{llll}27 a: & \text { 2n }: & a \propto A D \text { ad } & \text { MET met ur ur } \\ 27 b: & \text { 2n }: & a \propto A D \text { ad MET met UR UR } \\ 27 c: & \text { 2n }: & a \propto \text { ad ad MET met UR ur } \\ 27 d: & \text { 2n }: & a \propto A D A D M E T \text { met UR ur }\end{array}$

These genotypes agree with the observations on the phenotypes of the four segregants. They suggest that ascus 27 had originated from a tetraploid cell of the formula :

\section{4n : a a a a $A D A D$ ad ad MET MET met met UR UR ur ur}

The finding of a majority of regular asci in $C_{96} 6$ suggests that this hybrid (which had been grown from a single cell) was originally a diploid and that it became heterogeneous for ploidy subsequently. The formula suggested for the tetraploid mother cell of ascus 27 represents the double of the diploid genotype of hybrid $C 967$. Of the two mechanisms which could be responsible for such a doubling, namely, copulation of diploid cells and endomitosis, the latter would seem to be more probable since copulation figures have not been observed in diploid clones heterozygous for the mating type alleles.

A second ascus which gave unexpected segregations for most of the markers involved was obtained from hybrid $C 969$ :

\begin{tabular}{cccccc} 
Strain & Mating & \multicolumn{3}{c}{ Synthesis of } & Ferm. of \\
no. & type & Ad & His & Try & $G$ \\
$16 a:$ & $a$ & + & + & + & + \\
$16 b:$ & $a$ & + & - & - & + \\
$16 c:$ & $\alpha$ & + & + & + & + \\
$16 d:$ & $\alpha$ & + & + & + & +
\end{tabular}

Again, the observed ratios suggested segregation in a polyploid, and size and shape of the vegetative cells indicated that the four segregants were diploid. Since none of the clones could be induced to sporulate, segregants $a$ and $b$ (which in mass combinations with haploid testers behaved as $a$ strains) were crossed to segregants $c$ and $d$ (which in combinations with haploid testers reacted as $a$ strains). Abundant zygote formation was observed in all four crosses, $a \times c, a \times d, b \times c$, and $b \times d$. The four hybrids obtained from isolated zygotes resembled their parents in the shape of the cells but differed from normal diploids in that the cells were somewhat larger. All four hybrids sporulated abundantly, forming ascospores which were slightly larger than normal. The four hybrids were presumed to be tetraploids and 
expected to segregate into diploids. Segregations for the markers involved were studied in a series of asci isolated from the four hybrids. They were in agreement with the segregations expected from tetraploids if the diploid parents had been of the following constitutions :

$\begin{array}{llll}16 a: & 2 n: & \text { a a } A D \text { ad HIS his TRY TRY } G g \\ 16 b: & 2 n: & a\end{array}$

These genotypes are in agreement with the phenotypical segregations observed in ascus 16 . They indicate that the ascus originated from a tetraploid cell of genotype :

$4^{\text {n : }}$ a a a a AD AD ad ad HIS HIS his his TRY TRY try try GGg g, a constitution which represents the double of the diploid formula.of hybrid $C 969$. For the origin of the tetraploid mother cell, the same arguments apply as in the case of ascus $C_{967 / 27}$ discussed above.

The detailed results of analysis of the aberrant asci $C_{967 / 27}$ and C $969 / 16$ will be published separately.

\section{SUMMARY}

I. Data on segregations of a number of characters (mating type, growth factor requirements, galactose fermentation, and growth habit) in Saccharomyces cerevisiae are presented.

2. On the basis of the $2: 2$ segregations obtained, lin':age relations are calculated.

3. Complex segregations for galactose fermentation, pigment formation in adenineless strains, and uracil requirement, are interpreted in terms of dihybrid inheritance.

4. Two cases of complex segregations are shown to be due to tetraploidy.

Acknowledgments. - The authors wish to thank Dr B. Ephrussi for his aid in the direction of the work and his criticism during the preparation of the manuscript. We are further indebted to Dr $\mathbf{H}$. Roman for critical reading of the manuscript, and to Miss G. Burtin for technical assistance. The work was carried out while the senior author was the holder of a fellowship of the Swiss Foundation for Fellowships in Biology and Medicine.

\section{REFERENCES}

ADAMS, A. M. 1949. A convenient method of obtaining ascospores from bakers' yeast. Can. F. Res., C, 27, 179-189.

CAPUTTO, R., LELOIR, L. F., GARDIN, C. E., AND PALAdINI, A. C. I950. Isolation of the coenzyme of the galactose phosphate-glucose phosphate transformation. 7. Biol. Chem., 184, 333-350.

CAPUTTO, R., LElOIR, L. F., TRUCCO, R. E., CARDINI, C. E., AND PALAdINI, A. C. 1949. The enzymatic transformation of galactose into glucose derivatives. $\mathcal{F}$. Biol. Chem., 179, 497-498.

CAR DINI, C. E., PALADIN, A. C., CAPUTTO, R., LELOIR, L. F., AND TRUCCO, R. E., I949. The isolation of the coenzyme of phosphoglucomutase. Arch. Biochem., 22, 87-100. 
EPHRUSSI, B., AND hotTINGUER, H. I95I. Cytoplasmic constituents of heredity. Cold Spring Harbor Symp. Quant. Biol., 16, 75-85.

EPHRUSSI, B., HOTTINGUER, H., AND TAVLITZKI, J. 1949. Action de l'acriflavine sur les levures. II. Etude génétique du mutant "petite colonie". Ann. Inst. Past., 76, 419-450.

GILLILAND, R. B. 1949. A yeast hybrid heterozygotic in four fermentation characters. Ctes rend. Lab. Carlsberg, Serr. Physiol., 24, 347-356.

LeUPOLD, U. 1950. Die Vererbung von Homothallie und Heterothallie bei Schizosaccharomyces Pombe. Ctes rend. Lab. Carlsberg, Str. Physiol., 24, 381-480.

LEUPOLD, U. Tetraploid inheritance in Saccharomyces. (In press.)

Lindegren, c. c. I949a. Chromosome maps of Saccharomyces. P. Int. Gen. C. (8). pp. 338-355.

LINDEgren, c. c. 1949b. The Teast Cell, its Genetics and Cytology. Educ. Pub., Incorporated, St Louis.

LINDEGREN, c. C. 1953. " Concepts of gene-structure and gene-action derived from tetrad analysis of Saccharomyces. Experientia, 9, 75-80.

LINDEGREN, c. C., AND LINDEGReN, G. 1947. Depletion mutation in Saccharomyces. P.N.A.S., 33, 314-318.

LINDEGREN, c. C., AND LINDEGREN, G. I95I. Tetraploid Saccharomyces. F. Gen. Microbiol., 5, 885-893.

Lindegren, c. C., AND lindegren, G. 1952. Proximity of genes controlling the fermentation of similar carbohydrates in Saccharomyces. Nature, $170,965-968$.

MUNDKUR, B. D. 1949. Evidence, excluding mutations, polysomy, and polyploidy, as possible causes of non-Mendelian segregations in Saccharomyces. Ann. Miss. Bot. Garden, 36, 259-280.

MUNDKUR, B. D. 19.50. Irregular segregations in yeast hybrids. Current Sci., 19, $84-85$.

Papazian, н. G. 1952a. The analysis of tetrad data. Genetics, 37, 175-188.

Papazian, н. G. I 9526 . Unordered tetrads. Amer. Nat., 86, I 17 - I 18.

PERKINs, D. D. I949. Biochemical mutants in the smut fungus Ustilago maydis. Genetics, 34, 607-626.

PERKINs, D. D. 1953. The detection of linkage in tetrad analysis. Genetics, 38, I87-197.

POMPER, S. 1952. Isolation of triploid Saccharomyces cerevisiae. Nature, 170, 892-893. POMPER, S., AND BURKHOLDER, P. R. 1949. Studies on the biochemical genetics of yeast. P.N.A.S., 35, 456-464.

ROMAN, H., haWTHORNE, D. C., AND DOUglas, H. C. 1951. Polyploidy in yeast and its bearing on the occurrence of irregular genetic ratios. P.N.A.S., 37, 79-84.

SPIEGELMAN, S. 1952. Mapping functions in tetrad and recombinant analysis. Science, $116,510-512$.

TAvirtzkI, J. I95I. Sur les conditions de la formation de pigment chez une levure rouge. Rev. Can. Biol., 10, 48-59.

THORNE, R. S. W. 1951. The genetics of flocculence in Saccharomyces cerevisiae. Ctes rend. Lab. Carlsberg, Str. Physiol., 25, $101-140$.

TRUCCO, R. E., CAPUTTO, R., LeLOIR, L. P., AND MTTtELMAN, N. 1948. Galaktokinase. Arch. Biochem., 18, 137-146.

WICKERHAM, L. J. 1946. A critical evaluation of the nitrogen assimilation tests commonly used in the classification of yeasts. F. Bact., 52, 293-30I.

WTCKerham, L. J., AND BURTON, K. A. 1948. Carbon assimilation tests for the classification of yeasts. 7. Bact., $56,363-371$.

Whiterouse, H. L. K. 1950. Mapping chromosome centromeres by the analysis of unordered tetrads. Nature, 165,893 .

WINGE, ס., AND LAUSTSEN, o. 1937. On two types of spore germination, and on genetic segregations in Saccharomyces, demonstrated through single-spore cultures. Ctes rend. Lab. Carlsberg, Str. Physiol., 22, 99-1 16. 
WINGE, ø., AND ROBERTS, C. 1948. Inheritance of enzymatic characters in yeasts, and the phenomenon of long-term adaptation. Ctes rend. Lab. Carlsberg, Sér. Physiol., 24, 263-315.

WINGE, Ø., AND ROBERTS, C. 1950a. Non-Mendelian segregation from heterozygotic yeast asci. Nature, $165,157$.

WINGE, Ø., AND ROBERTs, c. 1950 $b$. The polymeric genes for maltose fermentation in yeasts, and their mutability. Ctes rend. Lab. Carlsberg, Sér. Physiol., 25, 35-83.

WINGE, Ø., AND ROBERTs, c. 1952. The relation between the polymeric genes for maltose, raffinose, and sucrose fermentation in yeasts. Ctes rend. Lab. Carlsberg, Ser. Physiol., 25, 141-171. 